\title{
Lidil
}

Revue de linguistique et de didactique des langues

$58 \mid 2018$

L'enseignement et l'apprentissage de l'écrit académique à l'aide de corpus numériques

\section{L'exploitation des corpus numériques dans les formations d'anglais scientifique en ligne : une étude de cas}

A Marriage of Reason between EAP and DDL: A Case of Blended Learning

Natalie Kübler et Clive E. Hamilton

\section{OpenEdition}

Journals

Édition électronique

URL : http://journals.openedition.org/lidil/5155

DOI : $10.4000 /$ lidil. 5155

ISSN : 1960-6052

Éditeur

UGA Éditions/Université Grenoble Alpes

Édition imprimée

ISBN : 978-2-37747-064-8

ISSN : $1146-6480$

Référence électronique

Natalie Kübler et Clive E. Hamilton, «L'exploitation des corpus numériques dans les formations

d'anglais scientifique en ligne : une étude de cas », Lidil [En ligne], 58 | 2018, mis en ligne le 02 novembre 2018, consulté le 01 mai 2019. URL : http://journals.openedition.org/lidil/5155 ; DOI 10.4000/lidil.5155

Ce document a été généré automatiquement le 1 mai 2019.

(C) Lidil 


\title{
L'exploitation des corpus numériques dans les formations d'anglais scientifique en ligne : une étude de cas
}

\author{
A Marriage of Reason between EAP and DDL: A Case of Blended Learning
}

Natalie Kübler et Clive E. Hamilton

\section{Introduction}

1 Depuis une dizaine d'années, la nécessité absolue d'enseigner l'anglais de spécialité comme atout professionnalisant dans les universités françaises fait consensus (Van der Yeught, 2014). Cependant, et c'est encore Van der Yeught qui le souligne, l'homogénéité de ce que l'on appelle le secteur LANSAD (Langues pour spécialistes d'autres disciplines) n'est qu'apparente. Les programmes et les approches mises en œuvre dans le secteur sont en effet assez hétérogènes, ce qui est dû en partie à la difficulté de fédérer un secteur mal reconnu par rapport aux secteurs disciplinaires en LLCE et LEA, et en partie au fait que le LANSAD s'organise de manière très différente selon les établissements (Braud, Millot, Sarré \& Wozniak, 2015 ; Brudermann, Mattioli, Roussel \& Sarre, 2016) : LANSAD centralisé dans un service langues ou un centre de ressources en langues, LANSAD comme département d'une UFR de langues ou d'une UFR LEA, LANSAD réparti par UFR disciplinaires, sans coordination entre enseignants LANSAD de celles-ci. En outre, le LANSAD est l'un des secteurs qui emploient le plus grand nombre de personnels non titulaires ayant des statuts très variés (lecteur, maître de langue, contractuel enseignant, vacataire, tuteurs étudiants, ATER), ce qui rend la spécialisation et la pérennité des programmes très complexes. Enfin, il s'agit d'un secteur qui recrute essentiellement des PRAG/PRCE et beaucoup moins d'enseignants-chercheurs ( $7 \%$ dont $1 \%$ de PU selon Brudermann, Mattioli, Roussel et Sarre (2015)). Par conséquent, les pouvoirs décisionnaires en ont une représentation biaisée. En effet, pour la grande majorité des 
instances universitaires, le LANSAD ne s'inscrit pas dans la recherche, car il s'agit uniquement pour celles-ci d'enseigner les langues aux spécialistes d'autres disciplines, une activité souvent considérée comme ancillaire, ce qui, comme le soulignent à juste titre Brudermann, Mattioli, Roussel et Sarre (2015, parag. 24), entre en contradiction avec les mission d'enseignement de l'université qui doit adosser son enseignement à la recherche. En outre, dans un domaine dans lequel les besoins sont aussi immenses, tirer parti des résultats d'une recherche dynamique permettrait sans doute d'améliorer l'offre de formation.

2 Or, et malgré les aprioris constatés dans le monde universitaire, le LANSAD a développé depuis la fin du siècle dernier une recherche dynamique et foisonnante tant dans le domaine de la didactique des langues que dans celui de l'anglais de spécialité proprement dit, ainsi que dans d'autres langues de spécialité (comme en témoignent les associations du GERES pour l'espagnol et du GERALS pour l'allemand), ainsi que toute la recherche développée depuis longtemps en didactique du FLE et, plus récemment, en FOU (français sur objectifs universitaires) et FOS (français sur objectifs spécifiques). Dans le monde anglophone, la recherche en ESP (English for Specific Purposes) et EAP (English for Academic Purposes) s'est développée depuis plus d'une trentaine d'années, notamment grâce à l'apport de la linguistique de corpus et des possibilités que celle-ci offre à la fois pour l'analyse et la description proprement dites des différents genres textuels (Swales, 1990), mais aussi pour l'apprentissage sur corpus (qui est l'équivalent français proposé par Boulton et Tyne (2014) du terme data-driven learning de Johns (1991)). Un nombre croissant d'études ont permis de mettre en exergue les spécificités de l'anglais universitaire, sous des angles multiples : voir, entre autres, Hardy et Römer (2013); Hyland (2000) ; Poudat et Follette (2012) ; Bordet (2015) pour les variations disciplinaires ; Clyne (1987) ; Fløttum, Dahl et Kinn (2006) ; Hartwell et Jacques (2014) pour les variations entre langues; Biber, Conrad, Reppen, Byrd et Helt (2002) ; Carter-Thomas et RowleyJolivet (2001) ; Hamilton et Carter-Thomas (2017) pour les variations entre langue orale et écrite en anglais scientifique. Ces traits caractéristiques symbolisent, en quelque sorte, le noyau dur ou l'ultime compétence à acquérir, par celui qui souhaite intégrer une nouvelle communauté de discours (Swales, 1990, 2016).

3 Cependant, ces traits spécifiques ne sont pas toujours pris en compte dans l'enseignement des langues de spécialité, comme le soulignent Chaplier et O'Connell (2015). En outre, les besoins en LANSAD devraient se décliner en fonction d'une analyse tenant compte des domaines et des situations de communication comme le plaident Nesi (2015) et Van der Yeught (2014). Celui-ci souligne par exemple les différences culturelles et institutionnelles dans le domaine du droit ou celui de la comptabilité entre la France et les pays anglophones. Une maitrise fine de la langue du droit en anglais exige que l'apprenant acquière des compétences juridiques dans la common law, qui diffère fortement du droit français par exemple. On peut penser que dans les disciplines scientifiques, ce type de problème ne se pose pas, mais d'autres difficultés se présentent à l'apprenant francophone. Enfin, les besoins communicationnels peuvent être variés, par exemple, rédiger un article scientifique en anglais, négocier un contrat, prendre la parole dans une réunion, rédiger un rapport d'activité d'une grande entreprise, passer un entretien d'embauche au téléphone. Toutes ces situations de communications sont liées à des communautés de discours qui se reconnaissent dans des genres textuels spécifiques que nos étudiants doivent apprendre à maîtriser, afin d'être légitimés dans les 
communautés de discours. Or, Swales (2016) revient sur cette notion pour l'élargir bien au-delà de la simple communauté de spécialistes d'un domaine.

Bien que de nombreuses études récentes aient abordé le problème du contenu à enseigner en LANSAD, Brudermann, Mattioli, Roussel et Sarre (2016), Faure (2013), Wozniak et Millot (2015), Chaplier et O'Connell (2015) et Van der Yeught (2014) indiquent que le problème principal résiderait dans le manque de formation des intervenants qui doivent préparer ces contenus faisant appel à une grande variété de situations de communications, et donc de genres dans des domaines spécialisés, sans pour autant qu'ils aient des connaissances antérieures propres à ces genres, ou même, à la notion de langue de spécialité. À ce propos, l'identification des obstacles à surmonter, de ce fait, n'est plus à refaire. Nonobstant, force est de constater que la mise en place de changements pédagogiques nécessaires s'installe très lentement en raison du fait qu'elle appelle davantage de moyens humains et techniques (Delabroye, Kübler, Leyboldt \& Perret, 2011), d'une part, et parce que le soutien des politiques linguistiques institutionnelles locales n'est pas toujours présent, d'autre part.

Des enjeux numériques s'ajoutent désormais à toute réflexion didactique, dans un contexte d'accélération du processus d'internationalisation des formations. L'avènement et l'accessibilité accrue des grands corpus numériques en anglais ont créé un terrain propice aux expérimentations pédagogiques. Ainsi avons-nous vu ces dernières années une augmentation des appels à favoriser le data driven learning ou apprentissage basé sur corpus (voir Johns, 1991; Kübler \& Foucou, 2000; Boulton \& Tyne, 2014). Ces expérimentations ont donné lieu à de nombreux matériaux didactisés qui semblent désormais de plus en plus répandus. À titre indicatif, nous trouvons à l'interface du lexique et de la phraséologie, l'Academic Word List élaborée par Coxhead (2000), l'Academic Keyword List (Paquot, 2010), l'Academic Phrasebank de l'université de Manchester et le projet ARTES (voir Kübler \& Pecman, 2012), pour n'en lister que quelques-uns.

6 Toutefois, nous voyons apparaitre, de manière progressive, un discret appel à favoriser des corpus disciplinaires spécialisés. En effet, Hyland et Tse (2007) soulignent l'importance de l'élaboration d'un répertoire lexical disciplinaire en lieu et place d'un répertoire lexical transdisciplinaire, notamment pour ceux qui poursuivent un cursus d'anglais scientifique. Nesi (2015) souligne également le mérite de ces corpus spécialisés. Elle affirme ne plus avoir recours uniquement aux grands corpus pour la création de matériel pédagogique en anglais de spécialité, mais aussi à des corpus spécialisés, ce qui n'est pas une tendance sui generis. D'autres, comme Charles (2012, 2014), mettent en avant la nécessité d'amener les apprenants à collecter leurs propres corpus spécialisés, de manière à coïncider au plus près avec la réalité du terrain disciplinaire auquel ceux-ci seront confrontés.

7 Notre article s'intéresse donc à deux aspects : l'exploitation concrète de corpus spécialisés en cours d'anglais scientifique, d'une part, et le regard porté par les principaux intéressés, c'est-à-dire les étudiants, face aux recours grandissant de ces ressources numériques, d'autre part. Nous tenterons donc, à la fin de cette contribution, d'apporter un début de réponse à trois questions qui nous paraissent essentielles :

1. La langue de spécialité devrait-elle être introduite tôt dans la formation d'anglais des étudiants spécialistes d'autres disciplines?

2. L'exploitation des corpus spécialisés devrait-elle être intégrée dans ces formations?

3. À partir d'un cas précis, comment les enseignants et les étudiants peuvent-ils travailler concrètement avec ces corpus spécialisés? 
Sont détaillés, dans la section suivante, les deux dispositifs d'enseignements, mis en place pour étudier ces deux aspects principaux. Puis, nous abordons l'appropriation de ces nouvelles ressources à la lumière des retours anonymes des étudiants.

\section{Deux dispositifs d'enseignements expérimentaux}

\subsection{Le contexte institutionnel}

L'expérience a été menée dans le cadre de trois structures étroitement liées : l'université Paris Diderot, le Pôle d'élaboration de ressources linguistiques (PERL) et le Centre de formation des doctorants à l'insertion professionnelle (CFDIP) de la COMUE Université Sorbonne Paris Cité (USPC).

1. La création d'un service de ressources linguistiques partagées entre plusieurs établissements, au sein de la COMUE USPC, le PERL ${ }^{1}$, a fourni un cadre propice à l'expérimentation de nouvelles formations en langues: notamment la création de formations en langues intégralement en ligne ou en hybride (c'est-à-dire, qui se composent de modules mélangeant présentiel et distanciel). Les deux dispositifs, que nous examinons dans cette étude, ont ainsi été créés dans ce cadre et mis à disposition sur une plateforme Moodle ${ }^{2}$.

2. L'université Paris Diderot, par le biais d'un rapport d'une commission de langues, a reconnu en 2011 les prémices du recours aux langues de spécialité dans l'enseignement des langues. Elle est, de ce fait, ouverte à diverses expérimentations en langues de spécialité. Le premier dispositif d'anglais scientifique a été proposé à des étudiants de cet établissement inscrits en première année d'un cursus de master.

3. Le Centre de formation des doctorants à l'insertion professionnelle (CFDIP), comme le PERL, se veut un service partagé entre établissements d'USPC. Il a fourni l'ensemble des doctorants-participants qui ont suivi la formation proposée dans le deuxième dispositif d'anglais scientifique basé sur corpus.

\subsection{L'expérimentation}

Plusieurs paramètres sont identiques dans les deux groupes d'essai (mastérants et doctorants). Tout d'abord, les tâches d'évaluation sont au nombre de trois: une évaluation orale et deux évaluations écrites. Ces dernières se présentent sous la forme d'un abstract ou résumé scientifique que les participants doivent rédiger et soumettre dès la première séance. Cette rédaction est ensuite resoumise à la fin de la formation avec des modifications et corrections apportées par le participant, sans que ce dernier ait bénéficié d'un retour individuel de l'intervenant. Ces écrits permettent, après une analyse comparée détaillée des deux tâches, de rendre compte de la progression (ou de l'absence de progression) de chaque participant. L'évaluation orale intervient lors de la dernière séance et se présente sous la forme d'un exposé. Nous demandons également à l'ensemble des participants de créer leur propre corpus spécialisé, composé de 5 à 10 articles minimum, selon la thématique de recherche de chacun. Enfin, les participants bénéficient tous d'une démonstration du logiciel AntConc (Anthony, 2014).

11 Dans la lignée de Renouf (1997, p. 256) qui fait la distinction entre exploiting to teach et teaching to exploit, ou de Beeby, Ines et Sanchez-Gijon (2009) qui, au sujet de l'utilisation des corpus dans la formation des traducteurs distinguent entre learning to use corpora to 
translate et learning to translate using corpora, deux approches ont été expérimentées : la première exploite donc les corpus pour créer du matériau pédagogique, la second apprend aux étudiants comment utiliser les corpus pour produire en L2. Les deux sections suivantes décrivent ces approches, ainsi que les paramètres qui diffèrent selon les groupes.

\subsubsection{De la sensibilisation passive basée sur corpus : le premier dispositif}

12 Le premier dispositif ou le groupe $\mathrm{A}$ se compose de 12 étudiants inscrits en première année d'un master à l'interface entre la biochimie et la chimio-informatique (Master In Silico Drug Design: ISDD). Le cours d'anglais scientifique est obligatoire et fait, par conséquent, partie intégrante du parcours universitaire. L'approche pédagogique mise en avant auprès de ce groupe se veut transmissive ou directe, ce qui consiste à adopter une démarche d'explicitation du savoir à acquérir. Le public n'entreprend pas de recherche active et participe uniquement par le biais d'exercices d'entrainement. Le format s'inscrit de ce fait dans un cours de langue plutôt traditionnel, mais faisant appel à des ressources peu utilisées en général : les corpus.

13 Il convient en effet de souligner que l'ensemble des éléments exposés dans ce groupe provient de cinq corpus différents : un corpus d'apprenants francophones écrivant en anglais (D9_FLC) ${ }^{3}$; deux corpus d'écrits d'étudiants anglophones (voir British Academic Written English (BAWE) ${ }^{4}$, Louvain Corpus of Native English Essays (LOCNESS) $)^{5}$; un corpus d'articles scientifiques écrits par des anglophones, issu du projet Étude interdisciplinaire et interlinguistique du discours académique $(\text { EIIDA })^{6}$; et un corpus maison réalisé à partir de deux manuels anglais obligatoires au programme du master, et concernant la biochimie et la chimio-informatique. Ces cinq corpus ont été analysés pour des faits de langue récurrents, aux niveaux lexical, syntaxique et phraséologique, de manière à collecter du matériau pédagogique. Les résultats ont été présentés aux étudiants sous diverses formes, ce qui nous a notamment permis d'effectuer des exercices d'applications par la suite.

14 L'analyse morphosyntaxique des cinq corpus a été effectuée par le biais de deux logiciels gratuits: TxM et LancsBox. Les résultats de chaque corpus ont été comparés et utilisés comme supports de cours. Ces comparaisons concernent, de manière générale, les traits syntaxiques et phraséologiques récurrents générés dans le but de contraster l'emploi réel de l'anglais scientifique par les étudiants anglophones (BAWE, LOCNESS), des étudiants en anglais L2 (D9_FLC) et des chercheurs expérimentés en anglais L1 (EIDA). De plus, au niveau du lexique simple et de la terminologie spécialisée, des concordances ont été établies pour illustrer les emplois en corpus.

15 À titre d'illustration, parmi les éléments utilisés comme supports de cours se trouve une étude contrastive de l'adverbe indeed. Cette comparaison illustre un des types de variations lexicales repérées dans les différents corpus, au niveau de son emploi réel, variations auxquelles les étudiants francophones rédigeant en anglais devraient être sensibilisés. Plus précisément, à travers une normalisation de l'usage selon la taille des différents corpus, on constate (voir fig. 1) une surutilisation de l'adverbe indeed chez les étudiants francophones par rapport à l'usage fait par des anglophones. Le ratio est calculé sur la base de 10000 mots. Ainsi, la fréquence observée de cet adverbe est de 15 occurrences tous les 10000 mots chez les étudiants francophones (corpus D9_FLC), alors que cette fréquence s'élève à 1 sur $10000 \mathrm{chez}$ les étudiants anglophones (corpus 
LOCNESS) et presque 5 sur 10000 chez les chercheurs expérimentés en anglais L1 (corpus EIIDA). Ces illustrations en cours de langue ont pour objectif d'évaluer le niveau d'appropriation de ces traits discursifs distinctifs chez les étudiants, à travers une sensibilisation d'usage réel par opposition à un enseignement basé exclusivement sur la grammaire des adverbes, par exemple.

Figure 1. - Fréquence de indeed par 10000 mots.

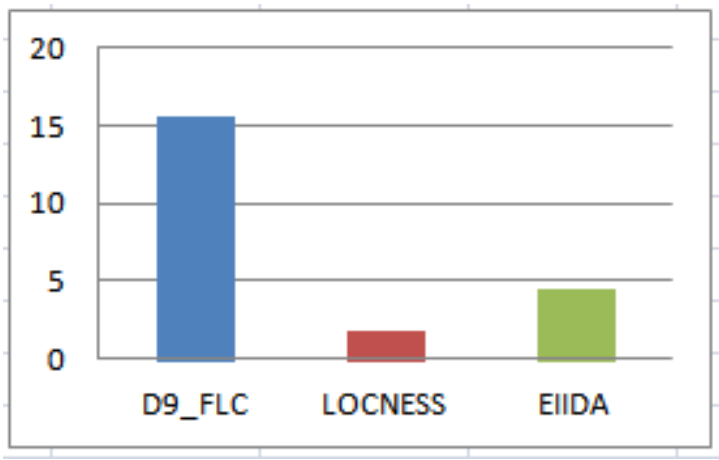

Un autre aspect lexical mis en lumière dans ce cours réside dans la prééminence observée de certains verbes dans le domaine de spécialité des étudiants. Ces observations proviennent du corpus établi à partir des manuels en biochimie et chimio-informatique. La liste extraite de ces manuels et fournie aux étudiants comporte les 185 verbes les plus fréquemment utilisés dans ces écrits disciplinaires. Nous estimons, par conséquent, que les étudiants doivent connaitre ces verbes et savoir les manier de manière appropriée, en respectant les schémas lexico-grammaticaux propres au domaine et à l'anglais scientifique. La figure 2 offre un aperçu des 30 premiers verbes issus du corpus, par ordre de fréquence. Dans ce dispositif, les étudiants bénéficient également d'informations relatives à la fréquence absolue de chaque verbe dans le corpus. De plus, cette liste contient des verbes appartenant à la fois à ce que l'on nomme le lexique scientifique transdisciplinaire ou la langue scientifique générale (voir Drouin, 2007; Tutin, 2007; Pecman, 2007) d'une part, et ce qui relèverait du lexique disciplinaire spécialisé (voir Coxhead \& Hirsch, 2007; Ha \& Hyland, 2017; Nation, Cohead, Chung \& Quero, 2016) d'autre part. Par exemple, parmi les 185 verbes attestés, seuls 99 apparaissent dans d'autres listes préalablement établies, comme par exemple l'Academic Keyword List. En effet, certains verbes, n'apparaissant sur aucune liste parmi celles que nous avons pu consulter, s'avèrent très fréquents dans le corpus disciplinaire : tels les verbes spécialisés to dock, to anneal dans lesquels le premier renvoie à une procédure de modélisation moléculaire, tandis que le deuxième insiste sur le caractère combinatoire de l'ADN. Le corpus spécialisé facilite ainsi le repérage de ces phénomènes terminologiques dont la connaissance est indispensable tant à l'enseignant d'anglais de spécialité, qui n'est pas forcément initié au domaine, qu'à l'étudiant apprenant l'anglais de sa discipline et qui est encore un apprenti dans celle-ci. 
Figure 2. - Fréquence verbale.

\begin{tabular}{|c|c|c|c|c|c|}
\hline 1 & use & 11 & find & 21 & determine \\
\hline 2 & identify & 12 & provide & 22 & give \\
\hline 3 & contain & 13 & see & 23 & take \\
\hline 4 & base & 14 & consider & 24 & dock \\
\hline 5 & show & 15 & describe & 25 & reduce \\
\hline 6 & calculate & 16 & include & 26 & know \\
\hline 7 & search & 17 & represent & 27 & predict \\
\hline 8 & select & 18 & generate & 28 & perform \\
\hline 9 & set & 19 & involve & 29 & correspond \\
\hline 10 & cluster & 20 & derive & 30 & follow \\
\hline
\end{tabular}

$\mathrm{Au}$ niveau syntaxique, en lieu et place des traditionnelles explications sur le fonctionnement grammatical du temps et de l'aspect dans la langue anglaise, nous avons abordé ces sujets à travers une comparaison de leur usage contextuel chez deux des trois groupes de locuteurs précédemment établis : à savoir les étudiants anglophones (L1) et les étudiants francophones (L2). Le tableau 1 illustre le résultat des comparaisons effectuées avec des variations d'emploi notables. Le tableau suggère, entre autres, que les étudiants francophones privilégient les conjugaisons au présent de l'indicatif là où les anglophones utilisent le prétérit ou past simple et le present perfect, par exemple.

Tableau 1. - Comparaison du temps grammatical.

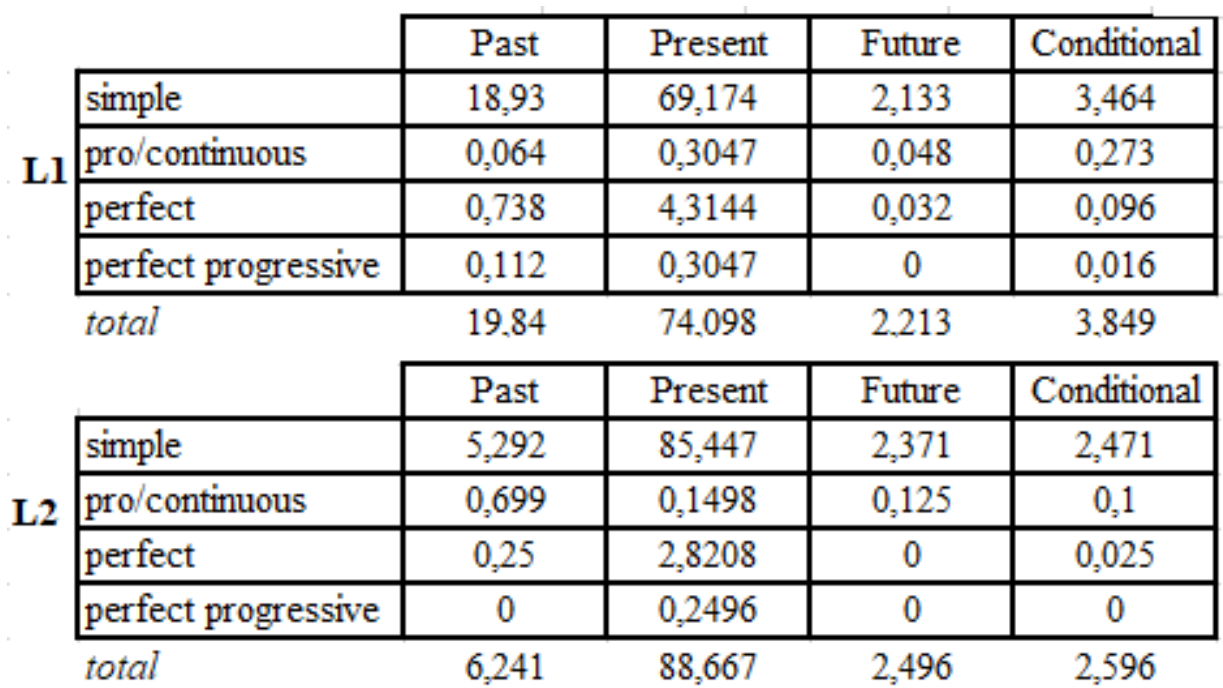

18 Ces comparaisons constituent le point d'entrée, dans ce cours expérimental, de l'étude du système temporel de la langue anglaise en anglais scientifique. D'autres aspects syntaxiques ont été abordés en partant du même prisme comparatif: à savoir, entre autres, l'utilisation de la voix passive dans les trois groupes de locuteurs, l'utilisation des pronoms personnels. De plus, la démarche comparative a également été utilisée afin d'introduire les éléments phraséologiques. 


\subsubsection{De la découverte participative : le deuxième dispositif}

19 Le deuxième dispositif ou groupe $B$ se compose de 20 doctorants de dix disciplines différentes, allant des sciences de l'éducation à l'imagerie médicale. Étant donné la nature du service proposé par le Centre de formation des doctorants à l'insertion professionnelle, les doctorants choisissent librement les enseignements qu'ils souhaitent poursuivre - souvent parmi un choix varié. Par exemple, plusieurs cours d'anglais scientifique sont proposés, mais un seul se veut hybride et basé sur corpus. Suivre cette formation relève, de ce fait, d'un choix assumé.

20 L'approche pédagogique diffère sensiblement de celle mise en place dans le groupe A. L'approche adoptée est celle de la découverte participative, déjà prônée par Holec (1990) et Johns (1991). Nous demandons aux doctorants un réel investissement, en ce qu'ils participent activement à la découverte du savoir. Les faits de langue ne sont pas analysés et présentés en amont. Ainsi, à travers des exercices variés, ils deviennent acteurs de l'ensemble des aspects qu'ils apprennent. En effet, ceux qui optent pour cette formation sont guidés dans leur exploration des corpus, de manière à identifier eux-mêmes les traits caractéristiques de l'anglais scientifique. Plus précisément, dès l'introduction de ce cours, sont présentés aux étudiants une vingtaine de jeux d'étiquettes, issus de TreeTagger, permettant d'interroger les corpus en ligne et de lancer des requêtes sur les catégories morphosyntaxiques.

21 Les corpus proposés à l'exploration proviennent essentiellement d'écrits scientifiques rédigés et publiés par des experts dans leurs domaines respectifs. Parmi les ressources exploitées, nous avons surtout utilisé le corpus EIIDA qui recouvre deux disciplines universitaires (à savoir la linguistique et la géochimie majoritairement, avec des articles écrits et leurs présentations orales correspondantes issues de plusieurs colloques). À cela s'ajoute un sous-ensemble d'articles de médecine extraits du Corpus of Contemporary American English (COCA). L'ensemble des corpus est hébergé sur une plateforme internet, du type CQPweb et est interrogeable pour des faits de langue touchant le lexique, la syntaxe, la phraséologie. La figure 3 montre un aperçu de la plateforme, telle qu'elle est présentée aux doctorants. Des gloses (par exemple LG = Linguistics) ont été ajoutées afin d'en faciliter la lecture. 
Figure 3. - Aperçu de la plateforme CQPweb.

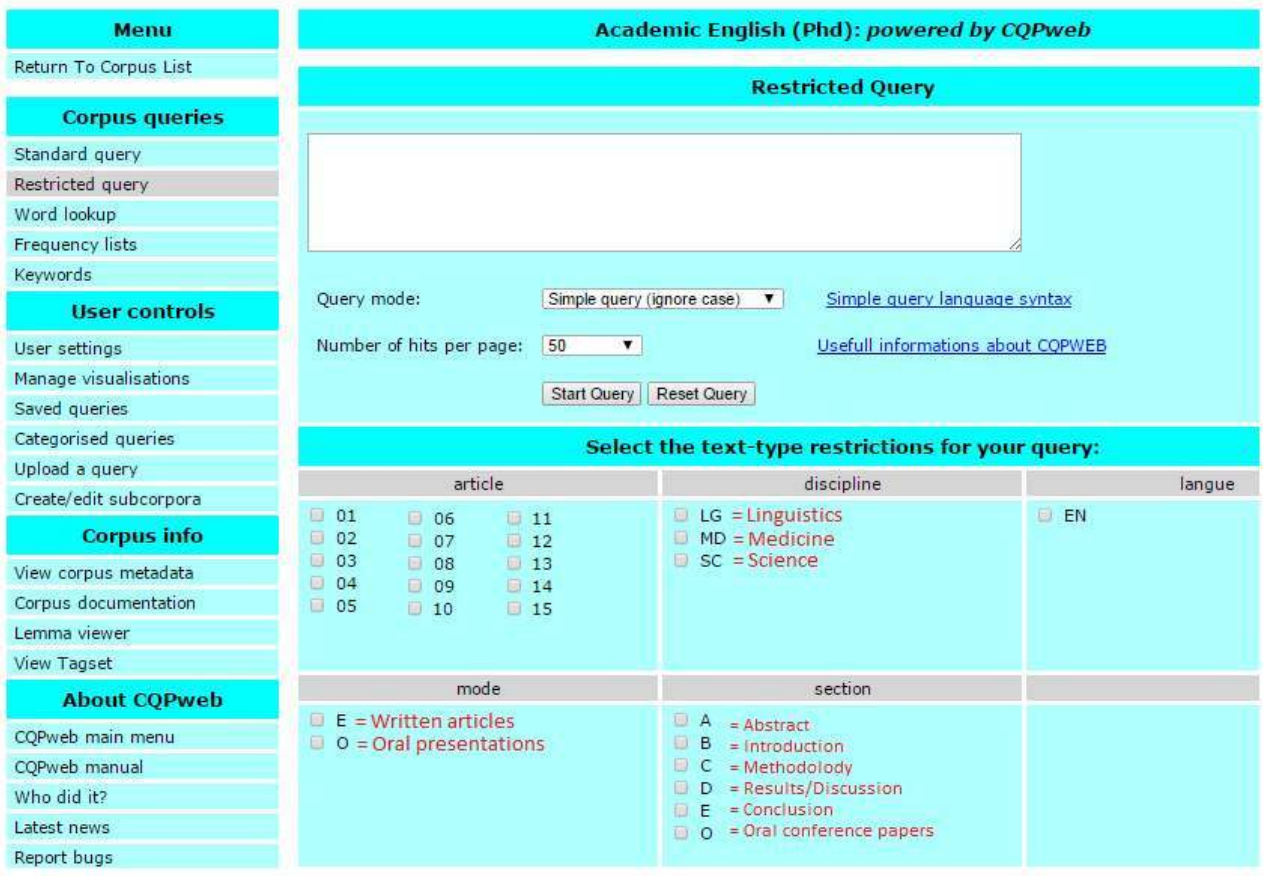

22 L'ensemble des éléments mis en avant dans le dispositif du groupe A est actualisé dans ce deuxième dispositif, à travers l'exploration des corpus. L'ancrage devient cependant davantage contextuel. Les aspects syntaxiques sont envisagés dans une perspective comparative entre les différentes sections de l'article scientifique. À titre d'illustration, nous demandons aux étudiants de comparer l'emploi de la voix passive et les temps grammaticaux dans l'abstract, l'introduction, la méthodologie et ainsi de suite, de manière à mettre en lumière les traits saillants propres à la rédaction scientifique. Ils découvriront alors, par exemple, que les structures passives représentent plus d'un tiers des groupes verbaux attestés dans une seule section de l'article entière - dans certaines disciplines alors que leur présence dans d'autres sections est rare.

Nous avons reproduit ci-dessous un exemple d'exercice permettant aux doctorants de repérer les différences d'emploi des temps dans les parties ${ }^{7}$ " introduction ", « méthode » et « discussion » des articles.

[...] examine the use of the present and past tense in sections B (introduction), $C$ (methods) and D (results and discussion) of the research paper. Use _VBP and _VBZ for present tense queries and_VBD for past tense queries. What do you notice? [...]

De surcroît, ce cours accorde une place importante, entre autres, à la terminologie et à la phraséologie simple et spécialisée. Les collocations y ont donc un rôle considérable. La figure 4, obtenue avec le logiciel AntConc, indique des concordances que les doctorants doivent obtenir eux-mêmes, lors d'un exercice spécifique, à partir du terme paper. Cette démarche permet aux doctorants, non seulement de visualiser les termes les plus usités dans les corpus spécialisés, mais également le contexte immédiat dans lequel lesdits termes sont employés. Autrement dit, à travers l'exploration des corpus, ils sont appelés à discerner et, par la suite, à s'approprier entre le « quand » et le « comment » des éléments interrogés. 


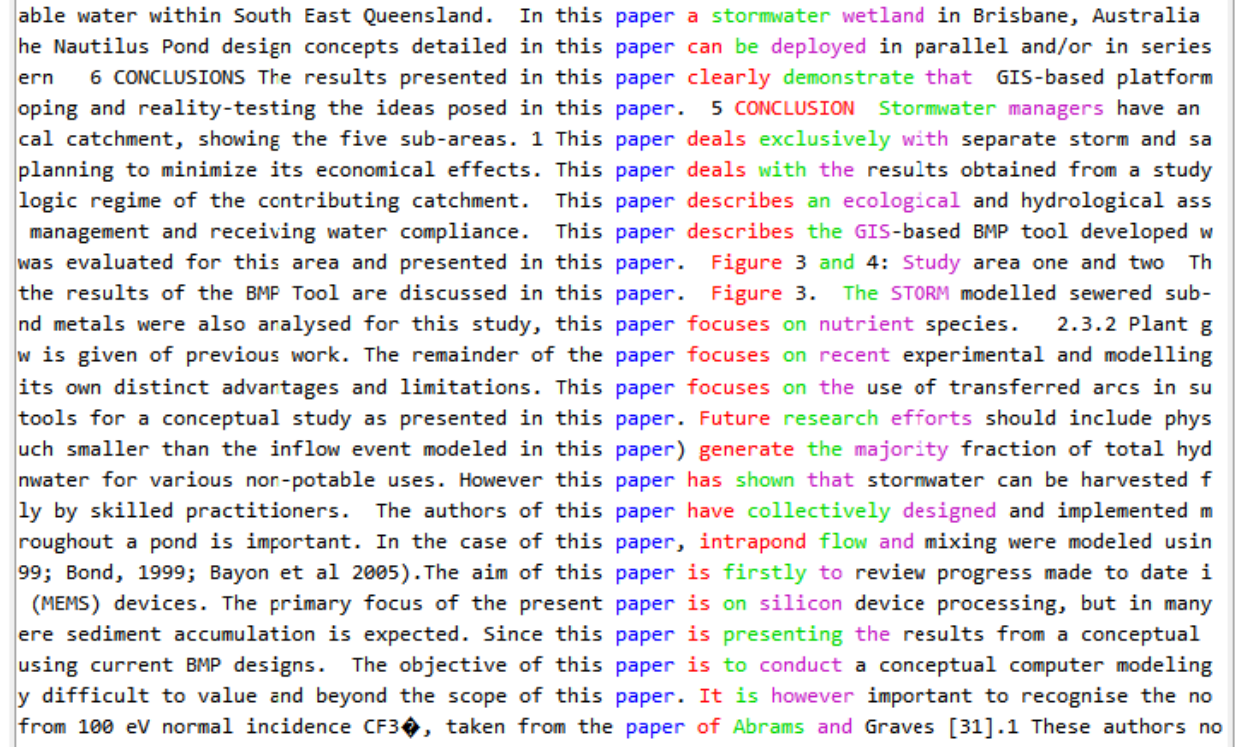

\subsubsection{Des enquêtes de satisfaction}

La mise en place de ces deux protocoles expérimentaux a été ponctuée par trois enquêtes courtes et anonymes dans le but de recueillir les ressentis du public visé par ces dispositifs. Trois questionnaires ont donc été intégrés à la plateforme pédagogique Moodle et soumis aux deux groupes en début, au milieu et à la fin de la formation. Le premier questionnaire portait principalement sur l'historique langagier des étudiants, leurs habitudes, leurs ressentis vis-à-vis des cours en ligne et leur utilisation générale de la langue anglaise. Les deuxième et troisième questionnaires se définissent comme des bilans de mi- et de fin de parcours respectivement, dans lesquels les étudiants se prononcent sur le format et le contenu aussi bien que sur les exercices et les ressources exploitées. Les participants se prononcent également sur le temps investi dans la formation, ainsi que les éléments qui leur ont semblé pertinents et soumettent, à la fin, leurs suggestions d'améliorations. Les résultats qui sont présentés dans la section suivante découlent de l'analyse de ces enquêtes.

\section{Le regard des apprenants sur les deux dispositifs}

\subsection{Un scepticisme latent à l'égard du cours en ligne}

Le premier questionnaire a permis d'identifier les différents niveaux de compétences des étudiants, face à l'outil informatique, qui rappelons-le, reste central aux deux dispositifs. Rappelons également que ces derniers se caractérisent par leur format hybride : c'est-àdire à la fois présentiel et distanciel. Chez les mastérants (groupe A), seulement $50 \%$ se considèrent à l'aise ou experts face à l'outil informatique. Notons que chez ces derniers, $66 \%$ déclarent passer plus de 4 heures par jour sur l'ordinateur ou la tablette informatique, tandis que ce chiffre s'élève à $94 \%$ chez les doctorants. De plus, dans le groupe B, $70 \%$ des participants se déclarent à l'aise voire utilisateurs experts, tandis que les $30 \%$ restant s'estiment des utilisateurs moyens. Ces éléments, à première vue 
négligeables, sont essentiels en ce qu'ils permettent d'évaluer la faisabilité et la pertinence des dispositifs face aux deux types de public. Ces premiers éléments tendent à montrer que l'outil informatique ne semble pas constituer un frein, en soi, au bon déroulement des cours, d'autant plus que $58 \%$ des doctorants et $73 \%$ des mastérants soutiennent avoir déjà suivi des cours en ligne.

Toutefois, deux constats supplémentaires appellent des remarques. De manière générale, sans avoir fait l'objet d'une certification en langues, $50 \%$ des étudiants en master estiment avoir un bon niveau dans les quatre compétences du CERCL en langue anglaise. Ce chiffre s'élève à $70 \%$ chez les doctorants. De ce fait, suivre une formation dispensée entièrement en anglais ne devrait pas, en principe, poser de problèmes conséquents pour une majorité d'étudiants. Nous avons cependant noté que deux-tiers des étudiants en master reconnaissaient ne pas faire de la recherche scientifique en anglais (c'est-à-dire consulter des articles scientifiques, etc.), ce qui peut influer, dans ce groupe, sur la réception ou l'attitude adoptée à l'égard du contenu exploité tout au long de cette formation. Signalons enfin que les étudiants des deux groupes ont émis un certain nombre de réserves à la fin du premier questionnaire. En effet, $31 \%$ du groupe $\mathrm{A}$ et $23 \%$ du groupe B ont estimé qu'un cours en ligne ne pouvait être aussi efficace qu'un cours dans une classe traditionnelle. Ces derniers ont cherché notamment à souligner le mérite de l'interaction, de l'oral et du suivi individualisé, face au défi du tout-informatisé.

\subsection{Un changement progressif de regard}

28 Le deuxième questionnaire intervient au milieu du semestre, de manière à obtenir des appréciations concernant la formation au fur et à mesure de sa progression. Le but initial de cette démarche était double: instaurer une sorte de contrôle qualité (c'est-à-dire, permettant d'évaluer, régler et adapter le cours en temps réel, le cas échéant) pour ensuite procéder à son amélioration générale pour les prochaines cohortes. En effet, hormis les questions portant sur les considérations techniques (ergonomie, clarté des consignes, des objectifs, des explications), cette deuxième enquête se concentre essentiellement sur des points ayant trait à l'évaluation du contenu linguistique.

Ce bilan de mi-parcours s'est révélé assez positif, dans la mesure où nous avons relevé une diminution considérable des réserves émises par des étudiants. C'est ce que l'on peut observer dans la figure 5 qui illustre leurs représentations globales exprimées en pourcentage. Les participants des deux groupes se rejoignent majoritairement sur la nature innovante, intéressante et dynamique du cours. Il en ressort toutefois que le dispositif mis en place dans le groupe A n'est pas jugé suffisamment dynamique par $30 \%$ du public. Cela incite à réfléchir sur les possibilités existantes sur la plateforme Moodle qui permettraient d'augmenter les modalités interactives du cours. L'approche directe adoptée, qui consiste à fournir du matériau pédagogique issu des corpus, sans demander aux étudiants d'observer eux-mêmes, dans une approche inductive, est peut-être aussi à l'origine de ces réserves. D'une certaine manière, néanmoins, l'appréciation positive globale est encourageante. Une amélioration du dispositif reste cependant nécessaire, mais ce constat n'enlève rien à l'intérêt de cette approche pour l'enseignement de la langue de spécialité. 
Figure 5. - Évaluation globale de mi-parcours.

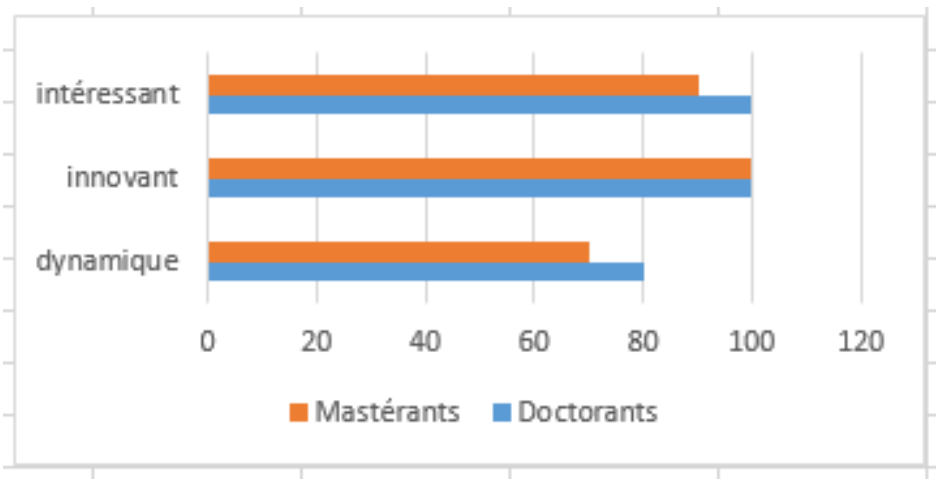

De plus, parmi les éléments évalués favorablement se trouve le fait que l'ensemble de la formation soit dispensé entièrement en anglais. Signalons que ce paramètre est remis en cause par $30 \%$ des étudiants en master et $20 \%$ d'inscrits en doctorat, notamment pour ce qui relève des explications théoriques (autrement dit, les explications proprement grammaticales ou linguistiques). Rappelons, à ce propos, que si la moitié des étudiants dans le groupe A et $70 \%$ dans le groupe B avaient affirmé avoir un bon niveau en anglais, ce n'était pas le cas pour les $30 \%$ restants, ce qui permet de mieux appréhender ce résultat. Par ailleurs, il est légitime de se demander s'il existe une corrélation entre les différences d'avis et les différents niveaux d'anglais des participants, mais, bien que cette hypothèse soit plausible, la nature anonyme et discontinue du premier et du deuxième questionnaire ne permet pas de la confirmer. Nonobstant, ce bilan de mi-parcours est jugé très important dans la mesure où les explications lexico-grammaticales doivent être suffisamment accessibles à l'ensemble des participants, de manière à assurer une certaine progression. Ainsi, il conviendrait de prendre en compte le niveau considérablement hétérogène, notamment au niveau master, et de trouver un moyen de faciliter la compréhension des explications théoriques, surtout si la formation continue à être dispensée entièrement en anglais.

Le deuxième questionnaire a permis de mettre en lumière une divergence de point de vue au niveau du contenu abordé, à proprement parler, dans les deux dispositifs. À la question «Pensez-vous qu'il manque des cours de grammaire?" l'ensemble des doctorants affirme ne pas regretter l'absence de cours de grammaire traditionnels (voir fig. 6).

Figure 6. - L'absence de grammaire.

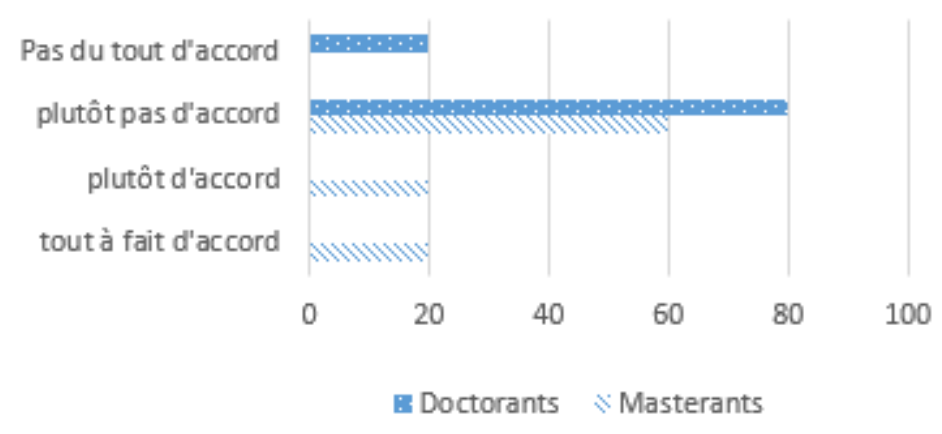




\begin{abstract}
aucun étudiant en master ne s'estime totalement convaincu par l'absence de ces cours. Il est alors possible d'inclure davantage de points de grammaire en s'appuyant sur les corpus, de manière à dynamiser cet aspect du cours et à aborder la grammaire d'une manière inductive.
\end{abstract}

à fait d'accord sur le fait qu'il manque des cours de grammaire traditionnels. De plus,
ant

\title{
3.3. Un investissement en décalage
}

Le dernier questionnaire soumis aux participants à la fin de la formation avait pour but de recueillir leurs avis globaux et leurs suggestions d'amélioration. Nous ferons néanmoins, ici, l'économie des remarques portant sur l'ergonomie, la fluidité et la gestion du contenu à proprement parler. En effet, à la différence de l'enquête singulièrement appréciative de mi-semestre, cette dernière est doublement nuancée. D'une part, la formation a été évaluée très positivement à l'intérieur d'un groupe, et d'autre part, l'apprentissage sur corpus a eu pour effet de pousser l'ensemble des participants à exploiter librement le potentiel des ressources mises à leur disposition pour effectuer une seule des deux dernières tâches demandées. Le décalage entre ces deux observations est examiné en détail ci-dessous.

En utilisant une échelle de Likert, les participants devaient se prononcer sur la question que nous reproduisons ci-après.

Pour ce qui est du contenu, le cours :

(i) vous a plu (échelle); (ii) vous a semblé utile (échelle); (iii) vous a demandé un investissement considérable (échelle); (iv) a suscité votre intérêt (échelle).

Les réponses des deux groupes sont illustrées en figure 7. Il ressort de la comparaison que l'ensemble des participants du groupe B estime que le cours à la fois leur a plu et a suscité leur intérêt. Dans le dispositif A, seulement 63 \% s'estiment satisfaits, d'une part, et $45 \%$ ont y trouvé un intérêt, d'autre part. Cette tendance globale à la baisse chez les mastérants doit, bien entendu, être analysée à la lumière des participants qui ont choisi de ne pas prendre position. Signalons qu'un écart d'investissement est également observé dans les deux groupes où $60 \%$ des doctorants soutiennent avoir consacré plus de 2 heures hebdomadaires et les $40 \%$ restant au moins $1 \mathrm{~h} 30$. D'un autre côté, seulement $40 \%$ des mastérants ont consacré plus de 2 heures hebdomadaires au cours. $40 \%$ affirment avoir passé moins d' $1 \mathrm{~h} 30$ et les $20 \%$ restant moins d' 1 heure hebdomadaire. Il semblerait donc qu'une corrélation existe entre les $60 \%$ qui ont consacré le moins de temps au cours et le $55 \%$ qui soutiennent que le cours n'a pas suscité leur intérêt. 
Figure 7. - Aperçu de la perception globale.

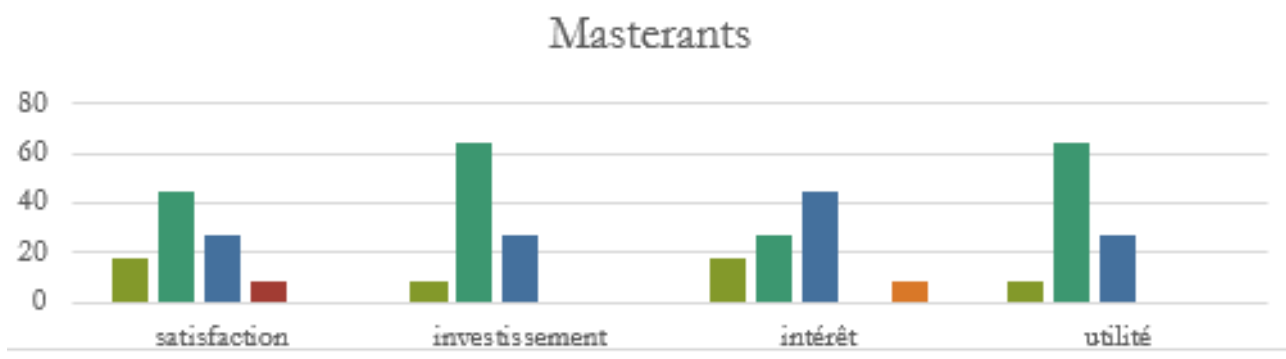

Doctorants

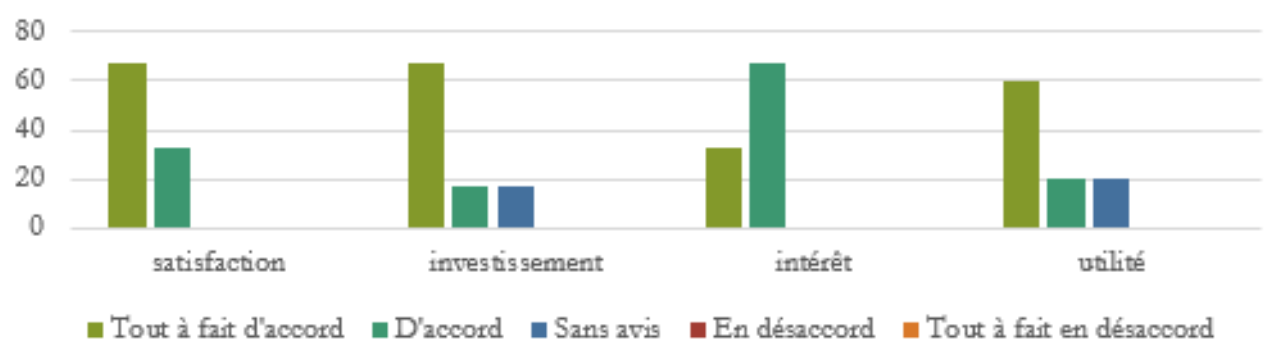

La dernière enquête a aussi permis de mettre en évidence une évaluation mitigée à l'égard de plusieurs autres points. De manière générale, les doctorants ont évalué l'ensemble des éléments positivement. Plus de $80 \%$ des effectifs, chez ces derniers, estiment que cet enseignement basé sur corpus leur a permis d'améliorer aussi bien l'expression écrite que la compréhension écrite. De surcroît, ils considèrent, à l'unanimité, que la focalisation sur les constructions syntaxiques et la phraséologie, mises en avant tout au long de la formation, leur a été bénéfique. Chez les mastérants, un nombre stable de participants s'est prononcé «sans avis ", ce qui ne permet pas de tirer de conclusions générales, au vu du faible nombre de participants dans chaque groupe. À la lumière de ces éléments, plusieurs questions s'imposent. Est-ce que l'évaluation positive dans le groupe B s'explique uniquement par rapport au fait que les doctorants accordent une plus grande importance à l'anglais scientifique que les participants dans le groupe A ? Ou est-ce en partie en raison du fait que le dispositif dans le groupe B est essentiellement participatif et demande une implication personnelle à chaque étape du cours?

Le dernier élément du questionnaire qui suscite des interrogations concerne l'utilisation des ressources. En effet, un ensemble de ressources a été mis à disposition des deux groupes tout au long de la formation. Pour le groupe A, ces ressources se limitaient aux transcriptions de plusieurs présentations scientifiques orales, issues du corpus EIIDA. Des références pour des corpus similaires (voir le Michigan Corpus of Academic Spoken English (MICASE), par exemple) ont également été fournies, de même que le logiciel AntConc. Pour le groupe B, il s'agissait de l'ensemble des corpus disciplinaires, les logiciels et les diverses transcriptions de présentations orales effectuées dans divers colloques.

Les retours du questionnaire ont démontré que les participants ont collectivement adopté une stratégie d'interrogation de corpus. En effet, les deux tâches finales dans les deux dispositifs consistaient en une rédaction scientifique et une présentation orale. Pour réaliser la rédaction, les participants des deux groupes affirment avoir utilisé des corpus d'articles qu'ils avaient compilé eux-mêmes, selon la spécificité de leurs recherches 
individuelles (voir section 2.2). À ces ressources se sont ajoutés les divers points abordés en cours. Cependant, les présentations orales n'ont pas bénéficié du même engouement pour les ressources du corpus, ce qui peut se justifier par l'attention principalement accordée aux phénomènes propres aux corpus écrits dans les deux dispositifs.

Nous considérons que le recours au corpus observé, dans la réalisation de la dernière tâche écrite, constitue un signe positif qui mérite d'être renforcé. En effet, malgré les divergences d'appréciation sur différents points mis en avant dans les deux dispositifs, la création et l'utilisation de petits corpus spécialisés semblent avoir conquis les deux publics. De plus, l'analyse comparée des deux tâches écrites (c'est-à-dire, les modifications et corrections effectuées par les participants entre la première et deuxième tâche écrite) permet de rendre compte des avantages réels de l'approche sur corpus.

\section{Conclusion}

Cet article a rendu compte de deux expérimentations menées dans des conditions originales pour l'enseignement de l'anglais scientifique. Bien que de nombreuses recherches soulignent les avantages de l'utilisation des corpus en anglais de spécialité, rares sont celles qui évoquent un enseignement sur corpus, hybride ou complètement en ligne. Ces expérimentations et leurs résultats nous amènent à émettre quelques hypothèses afin de répondre aux questions posées précédemment :

1. La langue de spécialité devrait-elle être introduite de manière précoce dans la formation LANSAD?

2. L'exploitation de corpus devrait-elle être intégrée dans ces formations?

41 Une prise de conscience précoce des différences entre langue générale et langue de spécialité, ainsi que langue universitaire, nous parait indispensable, d'autant plus qu'il s'agit de maitriser des caractéristiques linguistiques spécifiques, liées aux domaines de spécialité et aux genres textuels, dans une langue seconde, ce qui ajoute aux difficultés. La langue de spécialité nous parait donc pouvoir être abordée progressivement, dès la première année d'université, en tenant compte du niveau hétérogène en anglais des étudiants de première année. Parmi les réponses aux questionnaires, les étudiants de master ressentent le besoin d'avoir plus de grammaire. Nous plaidons en faveur de l'exploitation de corpus à la fois de langue générale et de langue spécialisée, mais aussi de langue semi-spécialisée (comme les textes de vulgarisation, qui ont recours aussi à des caractéristiques singulières) dans une approche active, de découverte de la grammaire, mais aussi de la phraséologie de l'anglais dès la première année d'université. L'objectif serait de faire progressivement prendre conscience aux étudiants des différences linguistiques entre langue générale et langue de spécialité, au-delà des questions de vocabulaire spécialisé. Par ailleurs, un deuxième objectif consisterait à leur faire acquérir le réflexe corpus, en s'appuyant tout d'abord sur les corpus disponibles en ligne, comme le Corpus of Contemporary American English, qui permet des requêtes différenciées sur la langue générale et la langue universitaire dans différents domaines. Par exemple, comme le montrent les deux expérimentations, un travail d'observation sur le passif ou le système temporel de l'anglais est nécessaire, ce qui fait partie de ce que les étudiants nomment "grammaire». Ce travail d'observation peut se faire à la fois sur un corpus général et de spécialité, ce qui fera ressortir l'emploi des temps selon le genre textuel par exemple. 

(Kilgarriff et coll. 2014), nous proposons d'apprendre aux étudiants, à partir de la troisième année d'université, à utiliser ces outils pour constituer leur propre corpus spécialisé, en fonction de leurs besoins. Les deux expérimentations ont en effet montré que les étudiants étaient capables d'utiliser spontanément les corpus pour accomplir certaines tâches. En outre, à partir du master, et en collaboration avec les enseignants des disciplines, il est possible de constituer avec ou sans l'aide des étudiants, des corpus spécialisés en lien avec les enseignements disciplinaires, de manière à permettre une acquisition plus efficace de la discipline en anglais.

Nous restons cependant conscients que des améliorations doivent être apportées au dispositif, en fonction des réponses des étudiants. Nous sommes aussi conscients de n'apporter qu'une pierre de plus à l'édifice enthousiaste que construisent depuis des années les chercheurs en linguistique de corpus pour l'enseignement et que la formation en anglais des spécialistes d'autres disciplines doit aussi s'appuyer sur une analyse des besoins et tirer parti d'autres approches (Sarré \& White, 2017, p. 6). Les deux approches présentées ici, à savoir, passive et inductive, exigent de l'enseignant un certain travail de préparation et une connaissance active de l'exploitation de corpus. En effet, soit l'enseignant compile des corpus qu'il interroge afin de constituer du matériau pédagogique servant de support de cours, soit il fait compiler leurs propres corpus aux étudiants, mais doit préparer des exercices d'entrainement pour amener les étudiants à poser les bonnes questions aux corpus, pour en reconnaitre les informations pertinentes. Ces quelques propositions nécessitent cependant que les instances décisionnaires acceptent de soutenir cette approche, mais aussi que les enseignants soient formés à l'exploitation des corpus. Nous espérons avoir montré comment des cas précis d'utilisation de corpus peuvent être mis en œuvre pour répondre à un besoin, c'est-à-dire, accomplir une tâche pédagogique représentant un besoin des étudiants spécialistes d'autres disciplines, tentant de répondre ainsi, comme de nombreux autres auteurs, à la question de savoir comment les enseignants et les apprenants peuvent travailler concrètement avec des corpus spécialisés.

\section{BIBLIOGRAPHIE}

ANTHONY, Laurence. (2014). AntConc (Version 3.4.4). Tokyo, Japon : Waseda University. Disponible en ligne sur <www.laurenceanthony.net/software>.

BEeby, Allison, InÉs, Patricia R. \& SÁNCHEZ-GIJón, Pilar (dir.). (2009). Corpus Use and Translating: Corpus Use for Learning to Translate and Learning Corpus Use to Translate (vol. 82). Amsterdam : John Benjamins Publishing.

Biber, Douglas, ConRAd, Susan, RePpen, Randi, Byrd, Pat \& Helt, Marie. (2002). Speaking and Writing in the University: A Multidimensional Comparison. TESOL Quarterly, 36, 9-48.

Boulton, Alex, CARTER-Thomas, Shirley \& Rowley-Jolivet, Elizabeth (dir.). (2012). Corpus-Informed Research and Learning in ESP: Issues and Applications (vol. 52). Amsterdam: John Benjamins.

Lidil, 58 | 2018 
Boulton, Alex \& TYNE, Henry. (2014). Des documents authentiques aux corpus : démarches pour l'apprentissage des langues. Paris : Didier.

BRAUD, Valérie, Millot, Philippe, SARRÉ, Cédric \& WozniaK, Séverine. (2015). « You say you want a revolution... » Contribution à la réflexion pour une politique des langues adaptée au secteur LANSAD. Recherche et pratiques pédagogiques en langues de spécialité, 34(1), 46-66. Disponible en ligne sur <http://apliut.revues.org/5020>.

Brudermann, Cédric, Mattioli, Marie-Annick, Roussel, Anne-Marie \& SARRÉ, Cédric. (2016). Le secteur des langues pour spécialistes d'autres disciplines dans les universités françaises : résultats d'une enquête nationale menée par la SAES. Recherche et pratiques pédagogiques en langues de spécialité, 35(1), 1-19. Disponible en ligne sur <http://apliut.revues.org/5564>.

CARTER-Thomas, Shirley \& Rowley-Jolivet, Elizabeth. (2001). Syntactic Differences in Oral and Written Scientific Discourse: The Role of Information Structure. ASp, 31-33, 19-37.

CHAPIER, Claire \& O'Connell, Anne-Marie. (2015). ESP and ASP in the Domains of Science and Laws in a French Higher Education Context: Preliminary Reflections. The European Messenger, 24(2), 61-76.

CHARLES, Maggie. (2012). "Proper Vocabulary and Juicy Collocations": EAP Students Evaluate DoIt-Yourself Corpus-Building. English for Specific Purposes, 31, 93-102.

CHARLES, Maggie. (2014). Getting the Corpus Habit: EAP Students' Long-Term Use of Personal Corpora. English for Specific Purposes, 35, 30-40.

Charles, Maggie, Pecorari, Diane \& Hunston, Susan (dir.). (2009). Academic Writing: At the Interface of Corpus and Discourse. Londres : Continuum.

CLYNE, Michael. (1987). Cultural Differences in the Organisation of Academic Texts: English and German. Journal of Pragmatics, 11, 211-247.

COXHEAD, Averil. (2000). A New Academic Word List. TESOL Quarterly, 34(2), 213-238.

COXHEAD, Averil. (2017). Approaches and Perspectives on Teaching Vocabulary for DisciplineSpecific Academic Writing. Dans J. Flowerdew \& T. Costley (dir.), Discipline-Specific Writing: Theory into Practice (p. 62-76). Londres : Routledge/Taylor \& Francis.

COXHEAD, Averil \& HIRSCH, David. (2007). A Pilot Science-Specific Word List. Revue française de linguistique appliquée, 12(2), 65-78.

Delabroye, Jean, KüBler, Natalie, Leyboldt, Edith \& Perret, Robert. (2011). Pour une politique des langues à l'université. Rapport interne de la Commission Langues, Université Paris Diderot.

DRouIN, Patrick. (2007). Identification automatique du lexique scientifique transdisciplinaire. Revue française de linguistique appliquée, 12(2), 45-64.

FAURE, Pascaline. (2013). Pour une appropriation de l'enseignement de la grammaire en secteur LANSAD. Les Langues modernes, 2013(1), 15-22.

FLøTtuM, Kjersti, DahL, Trine \& KinN, Torodd. (2006). Academic Voices: Across Languages and Disciplines. Amsterdam : John Benjamins.

GARDNER, Dee \& DAVIES, Mark. (2014). A New Academic Vocabulary List. Applied Linguistics, 35(3), 305-327.

GLEDHILL, Christopher \& KÜBLER, Natalie. (2016). What Can Linguistic Approaches Bring to English for Specific Purposes? ASp, 69, 65-95. 
HAMILTON, Clive E. (2015). Cartographie des erreurs en anglais L2 : vers une typologie intégrant système et texte (Thèse de doctorat, Université Sorbonne Nouvelle - Paris 3). Disponible en ligne sur < https://tel.archives-ouvertes.fr/tel-01378302> (consulté le 6 juin 2018).

HAmilton, Clive E. \& CARTER-Thomas, Shirley. (2017). Competing Influences: The Impact of Mode and Language on Verb Type and Density in French and English Scientific Discourse. Chimera : Romance Corpora and Linguistic Studies, 4(1), 13-34.

HA, Althea Y. H. \& HYLAND, Ken. (2017). What Is Technicality? A Technicality Analysis Model for EAP Vocabulary. Journal of English for Academic Purposes, 28, 35-49.

HARTWELl, Laura M. \& JACQUeS, Marie-Paule. (2014). Authorial Presence in French and English: "Pronoun + Verb" Patterns in Biology and Medicine Research Articles. Discours, 15. Disponible en ligne sur <http://discours.revues.org/8941>.

HARDY, Jack A. \& RoMËR, Ute. (2013). Revealing Disciplinary Variation in Student Writing: A MultiDimensional Analysis of the Michigan Corpus of Upper-Level Student Papers (MICUSP). Corpora, 8 (2), 183-207.

HoLEC, Henri. (1990). Des documents authentiques, pourquoi faire ? Mélanges pédagogiques, 65-74.

HYLAND, Ken. (2000). Disciplinary Discourses: Social Interactions in Academic Writing. Harlow :

Longman.

HYLAND, Ken \& TSE, Polly (2007). Is There an “Academic Vocabulary”? TESOL Quarterly, 41(2), 235-253.

Johns, Tim. (1991). Should You Be Persuaded: Two Examples of Data-Driven Learning. Dans T. Johns \& P. King (dir.), Classroom Concordancing, English Language Research Journal, 4, 1-16.

KilgaRRIfF, Adam, BAiSA, Vít, BušTA, Jan, JAKUBíčEK, Miloš, KovÁ̌̌, Vojtěch, Michelfeit, Jan, RYchlý, Pavel \& SUCHOMEL, Vít. (2014). The Sketch Engine: Ten Years On. Lexicography, 1, 7-36.

KÜBLER, Natalie \& Foucou, Pierre-Yves. (2000). A Web-Based Environment for Teaching Technical English. Dans L. Burnard \& T. McEnery (dir.), Rethinking Language Pedagogy: Papers from the Third International Conference on Language and Teaching (p. 65-74). Francfort-sur-le-Main : Peter Lang.

KÜBLER, Natalie \& PECMAN, Mojca. (2012). The ARTES Bilingual LSP Dictionary: From Collocation to Higher Order Phraseology. Dans S. Granger \& M. Paquot (dir.), Electronic Lexicography (p. 187-209). Oxford : Oxford University Press.

NATion, Paul, CoXHEAD, Averil, Chung, Mihwa \& QUERo, Betsy. (2016). Specialized Word Lists. Dans I. S. P. Nation, Making and Using Word Lists for Language Learning and Testing (p. 145-151). Amsterdam : John Benjamins.

NESI, Hillary. (2015). ESP Corpus Construction: A Plea for a Needs-Driven Approach. ASp, 68, 7-24.

PAQUOT, Magali. (2010). Academic Vocabulary in Learner Writing: From Extraction to Analysis. Londres et New York: Continuum.

Pecman, Mojca. (2007). Approche onomasiologique de la langue scientifique générale. Revue française de linguistique appliquée, 12(2), 79-96.

Poudat, Céline \& Follette, Peter. (2012). Corpora and Academic Writing: A Contrastive Analysis of Research Articles in Biology and Linguistics. Dans A. Boulton, S. Carter-Thomas \& E. RowleyJolivet (dir.), Corpus-Informed Research and Learning in ESP: Issues and Applications (p. 167-192). Amsterdam : John Benjamins. 
RENOUf, Antoinette. (1997). Teaching Corpus Linguistics to Teachers of English. Dans

A. Wichmann, S. Fligelstone, T. McEnery \& G. Knowles (dir.), Teaching and Language Corpora

(p. 255-266). Harlow : Addison Wesley Longman.

SARRÉ, Cédric \& WHYTE, Shona (dir.). (2017). New Development in ESP Teaching and Learning Research.

France : Researchpublishing.net

SWALES, John. (1990). Genre Analysis: English in Academic and Research Settings. Cambridge :

Cambridge University Press.

SwALES, John. (2016). Reflections on the Concept of Discourse Community. ASp, 69, 7-19.

Tutin, Agnès. (2017). Autour du lexique et de la phraséologie des écrits scientifiques. Revue

française de linguistique appliquée, 12(2), 5-14.

VAN DER YEUGHT, Michel. (2014). Développer les langues de spécialité dans le secteur LANSAD -

Scénarios possibles et parcours recommandé pour contribuer à la professionnalisation des

formations. Recherche et pratiques pédagogiques en langues de spécialité, 33(1). Disponible en ligne sur <http://apliut.revues.org/4153>.

\section{NOTES}

1. Voir le site internet USPC-PERL <https://perl2018.wixsite.com/perl-uspc>.

2. Voir le site internet MOODLE-PERL <http://perl.uspc.fr/>.

3. Ce corpus a été recueilli dans le cadre d'une thèse de doctorat, voir Hamilton (2015).

4. Le BAWE a été développé dans les universités de Warwick, Reading et Oxford Brookes, avec le financement de l'ESRC.

5. Le corpus LOCNESS a été réalisé par l'université catholique de Louvain.

6. EIIDA est un projet soutenu par le Labex TransferS, de l'École normale supérieure, Paris entre 2012 à 2015.

7. Ces parties correspondent à la structure prototypique des articles de recherche dite IMRAD : à savoir, Introduction, Méthode, Résultats et Discussion.

8. Le logiciel est désormais accessible gratuitement pour les établissements universitaires européens pour une période de quatre ans à partir de 2018. Voir le site du logiciel < www.sketchengine.co.uk>.

\section{RÉSUMÉS}

Cet article présente deux cas d'expérimentation concrète d'exploitation de corpus dans l'enseignement de l'anglais de spécialité universitaire, dans des formations hybrides. Nous adoptons deux approches: l'exploitation de corpus pour enseigner l'anglais de spécialité et enseigner aux étudiants à utiliser les corpus pour apprendre l'anglais de spécialité, dans une approche par tâches. Les étudiants ont répondu à des questionnaires dont l'analyse nous a permis de comparer leurs ressentis et représentations par rapport à une formation nouvelle pour eux, et leurs productions ont été analysées. Dans cet article, nous nous concentrons sur la présentation des expérimentations et sur l'analyse des questionnaires, afin de tenter de montrer 
concrètement comment exploiter les corpus dans des formations hybrides, mais aussi de tenter des propositions concrètes pour la formation en anglais des spécialistes d'autres disciplines à l'université.

This paper describes two task-based experiments using corpora for ESP and EAP blended learning. Two different approaches have been experimented: exploiting corpora to teach ESP and teaching to exploit corpora for ESP learning. In order to analyse the students' attitudes and representations towards this type of language course, questionnaires were submitted to the participants and their answers have been analysed. We will focus here on presenting the experiments and the questionnaire analysis. We aim to show how to use corpora in blended learning for EAP teaching. We will also make some suggestions for ESP training at university.

\section{INDEX}

Mots-clés : anglais scientifique, apprentissage hybride, apprentissage sur corpus

Keywords : academic English, blended learning, data-driven learning

\section{AUTEURS}

NATALIE KÜBLER

CLILLAC-ARP, Université Paris Diderot

\section{CLIVE E. HAMILTON}

CLILLAC-ARP, Université Paris Diderot 